\title{
Development of BIM-Based Safety Management Model Focusing on Safety Rule Violations
}

\author{
Idris Othman ${ }^{1, *}$, Mohamad Irwan P. Harahap ${ }^{1}$, Hisham Mohamad ${ }^{1}$, Nasir Shafiq ${ }^{1}$, and \\ Madzlan Napiah ${ }^{1}$ \\ ${ }^{1}$ Universiti Teknologi PETRONAS, Civil and Environmental Engineering Department, 32610 Seri \\ Iskandar, Perak, Malaysia
}

\begin{abstract}
Necessity of construction automation through BIM framework could no longer be set aside if we were to remain relevant and con-current in the ever-changing, technology-driven construction practices. The functions of construction safety management, i.e., hazard identification, assessment and control for construction works could certainly benefit from this technology through safety work automation which enhances the effectiveness and efficiency of the required safety processes. This paper aims to outline a research project for developing both information and technical requirements for BIM-based system via literature review, questionnaire survey, focus group, and prototype realization. The project results, to be validated by real projects, will act as solid platform for developing working model that can be employed at various project settings and safety circumstances. It will support the decision-making process with respect to safety enhancement and accident mitigation at the construction site.
\end{abstract}

\section{Background}

Public perception on the highly hazardous nature of the construction industry is for all practical purposes well founded based on the long records of accidents resulting in minor to serious injuries and even fatalities at the project site across the globe. For instance, according to Occupational Safety and Health Administration (OSHA) in 2016991 (21.1\%) worker fatalities were in the construction industry. To drive this point home, the agency also introduced the so-called "Fatal Four" causes of the accident namely falling from height (38.7\%), being struck by object (9.4\%), electrocution (8.3\%) and getting caught in between objects (7.3\%) [1]. Closer home in Singapore, the fatality has increased from 24 in 2006 to 33 in 2013 [2]. Clearly, the rise in construction accidents goes in tandem with incremental development within the respective country over the years.

In the same measure, the Malaysian construction industry is not immune from this dilemma. The Department of Occupational Safety and Health (DOSH) reported that as of October 2017 the construction industry contributed the most in terms of occupational deaths at 63 cases [3]. However, it is interesting to note that the same statistics indicated

*Corresponding author: idris_othman@utp.edu.my 
relatively low overall casualty count from the industry indicating the severity of the accident, frequency and job sector are intertwined. Furthermore, other study from 2005 to 2009 has attributed the causes of accidents at the construction site to stepping on, striking against or being struck by object (47\%), falling from height $(27 \%)$, getting caught in between objects $(9 \%)$, overexertion or strenuous movement $(4 \%)$ and other accident types $(13 \%)$ [4]. The trend generally pointed to an upward movement over the years from 3738 cases in 2008 to 4108 cases in 2009 (refer to Figure 1).

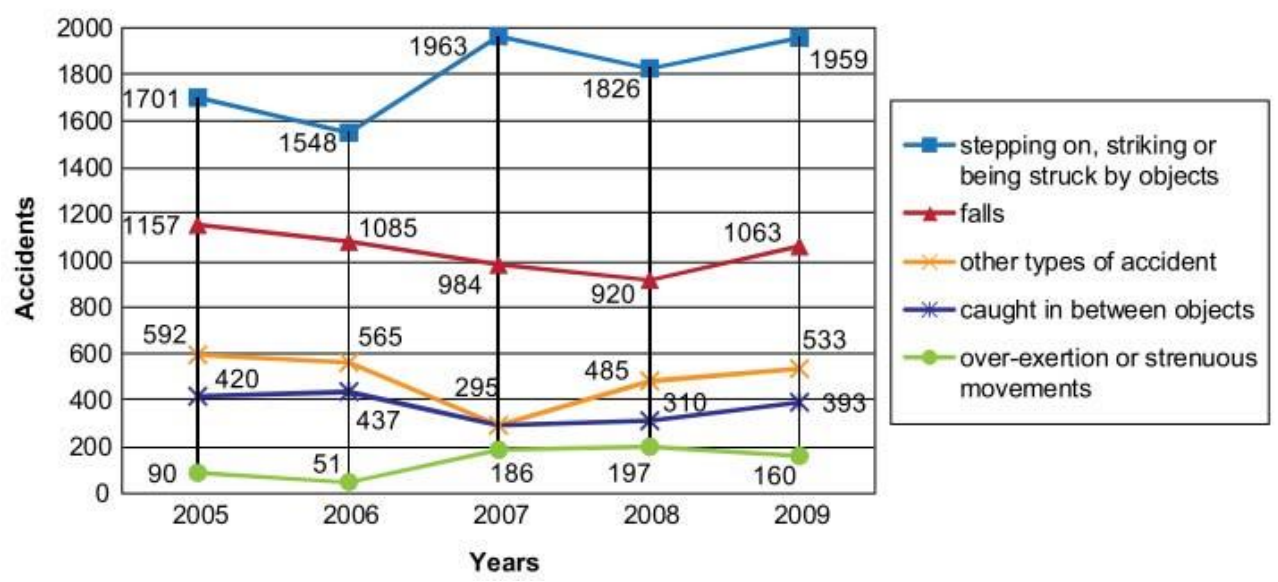

Fig. 1. Causes of accidents (2000-2009) [4].

\section{Safety Planning}

Treating safety issues in isolation from other construction practices were the norm in many projects in the past. Safety concern and observation were regarded inconsistent with the rough-and-tumble nature of the construction industry to the extent of hindering productivity and imposing unnecessary capital expenditures. As a result, accidents occurred in frequent basis but much of the unfavourable statistics were camouflaged to give a positive impression to the public. However, as the statistics grow and scrutinized by the relevant authorities, it becomes evident that accidents do directly influence cost, time and quality of the construction

Clearly, a well-integrated approach is needed in order to ensure smooth execution of the project throughout its life cycle which includes written safety plans [5]. No measure could adequately substitute for planning. Nevertheless, many constructors have found it difficult to convert Safety Management Regulations directive into actions and practical implementation. Furthermore, failure to identify hazards and risks could lead to accidents at the site. As such, developing good safety culture through observation of safety rules and regulations is essential. And BIM technology could facilitate in developing this mentality.

\section{BIM Applications in the Construction Industry}

Building Information Modelling (BIM) is a digital representation of a facility's physical and functional characters [6]. It allows for three-dimensional (3D) representation and integration of both geometric and semantic information obtained from architect, engineer, contractor and facility manager. Thus, it has the capability to facilitate transparent information sharing and management of facilities [7][8] and interactive operations for the clients or users through the entire project life cycle [6]. As a result, cross-specialty 
collaborative efforts and coordination would be greatly facilitated as a result, and ideas and designs could be tested for veracity and practicality well in advance of the actual implementation process. In addition, BIM can be further enhanced through providing additional dimensions such as cost, schedule, accessibility, safety, logistics, security, sustainability, maintainability, acoustics and energy simulation [9][10]. As such, many useful project information such as constructability, sequencing, value and engineering reports could be communicated systematically and efficiently to all project stakeholders at various project participatory levels and minuteness. Small wonder BIM should find ready acceptance from among the construction practitioners and has been in use to manage various aspects of the construction process and will continue to grow significantly in the coming years [11].

In order to gauge the extent of BIM penetration in the construction industry, a study has been carried out through pre-selected journal review and verified by the author which applies categorization based on BIM system deliverables [12]: procurement [13], sustainable design and analysis [14], safety design and management [15], design alternatives selection and optimization [16], operation and maintenance [17], supply chain management [18], construction planning and management [19], quality management [20], automated building design review [21], fabrication [22], information integration, management and visualization, [23], cost management [24] and commissioning [25]. The multi-faceted implementation and broad usage of BIM in the industry at present has indicated a high level of flexibility of the BIM infrastructure in transforming various aspects of the traditional management processes involving the planning, design, execution, operation and maintenance stages of the project. However, most of the quoted examples are restricted to systems primarily utilized and available in developed countries.

In the context of Malaysia, the unsettling tendency prevails in which more effort is placed on academic researches on the topic rather than real technology adoption by the industrial players [26]. This is not in line with the governments strive towards Industry 4.0 status in which automation of the construction sector would be some of the key areas to be prioritized in order to minimize reliance on foreign labours and associated problems. Some root causes need to be identified in order to reverse this undesirable outcome. Mamter et al disclosed that the absence of compulsive authority rulings, poor holistic readiness among stakeholders, software integration competition strategy, and resistance to change and knowledge-sharing has contributed significantly to the problem regardless of the obvious benefits and currency of such an adoption [27]. Progressive governmental intervention might be required to persuade or compel industrial players to subscribe to the BIM work process.

In the realm of safety management, the BIM technology in various guises has been available for a while. Hongling et al has reported the Design for Safety (DfS) approach in which BIM is integrated with design safety rules in order to identify potential safety problem [28]. However, this system could be abused due to reliance on fickle human factor under assumption that they would be observing safety rules and regulations consistently. Another parallel BIM-based framework to support safe maintenance and repair practices during the facility management phase through safety attribute identification/classification, data processing and rule-based decision making, and a user interface has been introduced by Wetzel et al [29] but since it is potentially intended to work within the last stage environment of the project life cycle the safety risk mitigation benefit could be minimal. The next reported study actually developed a prototype of automated safety rule-checking algorithms for BIM that simulates fall hazard detection and prevention, thus predicting and eliminating such hazard early in the planning phase of a construction project [30]. Here however the variability of independent factors could affect the prediction model. 
With the research gaps thus established, the author would like to propose a BIM framework that manages non-conformity or violation of safety rules and regulations as primary decision determinant whether accidents may or may not have occurred. This model would automate the construction safety management process, which includes artifacts (BIM components) embedded with selective safety-related properties or classes, with corresponding safety rules, scheduling information, geographic information, and so on including details of safety violation in their respective vicinity which are committed by site employees in their personal capacity. This would be a generic system that could be adapted to multiple site settings and safety circumstances, and a prototype would be developed and deployed at a working construction site to test this hypothesis and assess its performance.

\section{Details of Proposed Research}

The research project aims to develop a framework and prototype to manage safety issues at the construction site particularly during the construction execution stage. To achieve this aim, the objectives are:

1. To systematically establish cause and effect association between safety violation factors and accidents.

2. To develop BIM framework to manage safety violations issues t the construction site.

3. To develop prototype and assess its performance through a case study.

The above objectives will be realized by carrying out literature review, questionnaire survey, focus group study and prototype realization.

1. Literature Review - A comprehensive literature review will be conducted to define and refine the research framework (e.g., safety parameter, BIM adoption in safety, the identified benefits and barriers, potential software tools), and to develop survey and focus group interview protocol. The review will cover both academic and professional reports, journal papers and websites.

2. Questionnaire Survey - A questionnaire survey will be developed based on the extensive literature review to investigate the current BIM adoption level in different construction stages, and the anticipated critical factors and components that may influence the developed model, covering both informational and technical aspects. The survey study will target construction safety professionals (say 100 respondents involving safety managers, safety supervisors, safety officers, and/or assistant safety officers). The quantitative data collected will be analysed by statistical techniques, including factor analysis, reliability analysis, Pearson correlation and multiple regression modelling, using SPSS. In order to obtain high response rate, assistance may be sought from various professional bodies e.g. DOSH, CIDB, IEM, etc., to recruit survey respondents and focus group interviewees through their membership database.

3. Focus Group Study - Through providing and interactive platform for BIM experts and construction safety professionals to exchange knowledge and demonstrate their expertise in BIM and construction safety, the focus groups will be extremely effective in helping to understand the prevailing situation, perceived benefits and barriers of SIM in construction, and to identify information and technical requirements/logics of SIM. The qualitative data collected will be analysed by systematic contextual analysis. 
4. Prototype Realization (Case Study) - A prototype of the BIM framework would be developed, deployed and operated by the safety personnel from a pre-selected case study for a period of time (say 3 months). Then, Questionnaire Survey shall be administered to the affected personnel in order to gauge the performance of the system.

\section{Conclusions}

With the application of well-structured BIM-based safety management model focusing on the safety violations with the intention of further administrative follow-ups by the management team against the offenders, this could provide an effective and efficient deterrent towards addressing growing construction accidents at the site and systematic record keeping for future reference and conflict resolution that might have arisen.

\section{References}

[1] O. S. and H. A. OSHA, "Commonly Used Statistics," 2016. [Online]. Available: https://www.osha.gov/oshstats/commonstats.html.

[2] M. of M. MOM, "Statistics and Publications," 2017. [Online]. Available: http://www.mom.gov.sg/.

[3] D. of O. S. and H. M. DOSH, "Occupational Accidents Statistics by Sector Until October 2017," 2017. [Online]. Available: http://www.dosh.gov.my/index.php/en/occupational-accident-statistics/by-sector.

[4] H. Y. Chong and T. S. Low, "Accidents in Malaysian construction industry: Statistical data and court cases," Int. J. Occup. Saf. Ergon., 20 (3) pp. 503-513, 2014.

[5] M. Hallowell, "Cost-effectiveness of construction safety programme elements," Constr. Manag. Econ., 28, pp. 1, 25-34, 2010.

[6] X. Wang, P. E. D. Love, M. . Kim, C.-S. Park, C.-P. Sing, and L. Hou, "A conceptual framework for integrating building information modeling with augmented reality," Autom. Constr, pp. 34, 37-44, 2013.

[7] P. E. Wang, X.; Love, "Onsite information sharing and communication via advanced visualization," in IEEE 16th International Conference on Computer Supported CooperativeWork in Design (CSCWD, 2012, pp. 850-855.

[8] Y. Wang, X.; Truijens, M.; Hou, L.;Wang, Y.; Zhou, "Integrating augmented reality with building information modeling: Onsite construction process controlling for liquefied natural gas industry," Autom. Constr., pp. 40, 96-105, 2014.

[9] P. G. Taylor, J.E.; Bernstein, "Paradigm trajectories of building information modeling practice in project networks,” J. Manag. Eng., pp. 25, 69-76, 2009.

[10] S. Aouad, G.; Lee, A.;Wu, Constructing the Future: Nd Modelling. London, UK: Routledge, 2006.

[11]J. Young, Norbert W., S. A. Jones, and H. M. Bernstein, "Building Information Modeling: Transforming Design and Construction to Achieve Greater Industry Productivity," McGraw-Hill Construction, New York, 2008.

[12] W. Shou, J. Wang, X. Wang, and H. Y. Chong, "A Comparative Review of Building Information Modelling Implementation in Building and Infrastructure Industries," Arch. Comput. Methods Eng., 22, (2) pp. 291-308, Apr. 2015.

[13] A. Grilo and R. Jardim-Goncalves, "Challenging electronic pro- curement in the AEC sector: a BIM-based integrated perspective," Autom Constr, 20, pp. 107-114, 2011. 
[14] S. Azhar, W. Carlton, and D. et al Olsen, "Building information modeling for sustainable design and LEED rating analysis," Autom Constr, 20, pp. 217-224, 2011.

[15] S. Zhang, J. Teizer, J. K. Lee, C. M. Eastman, and M. Venugopal, "Building Information Modeling (BIM) and Safety: Automatic Safety Checking of Construction Models and Schedules," Autom. Constr., 29, pp. 183-195, 2013.

[16] S.-I. Lee, J.-S. Bae, and Y. Cho, "Efficiency analysis of set-based design with structural building information modeling (S-BIM) on high-rise building structures," Autom Constr, 23, pp. 20-32.

[17] A. Motamedi and A. Hammad, "RFID-assisted life cycle management of building components using BIM data," in 26th international symposium on automation and robotics in construction (ISARC 2009), 2009.

[18] J. Irizarry, E. Karan, and F. Jalaei, "Integrating BIM and GIS to improve the visual monitoring of construction supply chain management," Autom Constr, 31, pp. 241254, 2013.

[19]H. Li, T. Huang, and C. et al Kong, "Integrating design and construction through virtual prototyping," Autom Constr, 17, pp. 915- 922, 2008.

[20]E. Anil, P. Tang, and B. et al Akinci, "Deviation analysis method for the assessment of the quality of the as-is building information models generated from point cloud data," Autom Constr, 35, pp. 507- 516, 2013.

[21] J.-K. Lee, J. Lee, and Y.-S. et al Jeong, "Development of space database for automated building design review systems," Autom Constr, 24, pp. 203-212, 2012.

[22] Q. Moya and O. Pons, "Improving the design and production data flowof a complex curvilinear geometric glass reinforced concrete façade," Autom Constr,38, pp. 46-58, 2014.

[23] I. Wu and S.-H. Hsieh, "A framework for facilitating multi- dimensional information integration, management and visualization in engineering projects," Autom Constr,23, pp. $71-86$.

[24]Z. Ma, Z. Wei, and X. Zhang, "Semi-automatic and specification- compliant cost estimation for tendering of building projects based on IFC data of design model," Autom Constr, 30, pp. 126-135, 2013.

[25] W. Wu and R. Issa, "BIM-enabled building commissioning and handover. In: Computing in civil engineering," in Computing in civil engineering, 2012, pp. 237244.

[26] W. I. Enegbuma, G. U. Aliagha, and K. N. Ali, "Effects of perceptions on BIM adoption in Malaysian construction industry," J. Teknol.,77, no. 15, pp. 69-75, 2015.

[27] S. Mamter, A. R. Abdul-Aziz, and M. E. Mamat, "Stimulating a Sustainable Construction through Holistic BIM Adoption: The Root Causes of Recurring Low BIM Adoption in Malaysia," in IOP Conference Series: Materials Science and Engineering, 2017, 216 (1)

[28] G. Hongling, Y. Yantao, Z. Weisheng, and L. Yan, "BIM and Safety Rules Based Automated Identification of Unsafe Design Factors in Construction," in Procedia Engineering, 2016, 164, pp. 467-472.

[29]E. M. Wetzel and W. Y. Thabet, "The use of a BIM-based framework to support safe facility management processes," Autom. Constr.,60, pp. 12-24, 2015.

[30] S. Zhang, K. Sulankivi, M. Kiviniemi, I. Romo, C. M. Eastman, and J. Teizer, "BIMbased fall hazard identification and prevention in construction safety planning," Saf. Sci.,72, pp. 31-45, 2015. 